\title{
Component processes in the perception of bilaterally symmetric dot textures
}

\author{
BILL JENKINS \\ University of Western Australia, Nedlands, Australia
}

\begin{abstract}
If a random-dot texture is reflected about a given axis, the resulting bilateral symmetry is immediately detected. In order to account for this ability, it has been suggested that the visual system detects reflection in these dot textures because of the existence of a symmetric neural organization, centered about the fovea, which performs a point-by-point analysis. As yet, there is no evidence of such an organization in the human visual system. An alternative description of a bilaterally symmetric texture, that of a two-dimensional distribution of uniformly oriented point-pair elements, of nonuniform size and with collinear midpoints, is more consistent with known mammalian visual-system neurophysiology. Experiments were conducted in order to determine whether the human visual system is sensitive to the orientational uniformity of otherwise nonuniform point-pair elements and to their midpoint collinearity. The results indicate that there are three processes involved in the perception of bilaterally symmetric dot textures: the detection of orientational uniformity of the different sized point-pair elements; the fusion of salient element point-pairs into a salient feature; the detection of the symmetry of the resulting feature. This component-process hypothesis eliminates the necessity to postulate the existence of a symmetrical neural organization centered about the fovea.
\end{abstract}

If a random-dot texture is reflected about a given axis, the global pattern of the resultant bilaterally symmetric texture is immediately perceived. This immediacy of perception led Julesz (1971) to suggest that "for patterns with high spatial resolution, it seems that there is a point-by-point comparison process based on neural anatomy that has a symmetrical organization around the center of the fovea" (p. 131). In addition, to account for the salience of the information close to the axis of reflection, he postulated that this comparison was "strongly weighted in favor of areas close to the axes of symmetry"' (p. 131).

However, as yet, there is no direct neurophysiological evidence for such a symmetrical neural organization in the mammalian visual system. There is, however, evidence of an organization in the visual cortex, with well-defined functional properties, which comprises columns of like sensitive cells. A major aspect of this organization is that, within columns, the cells have similarly oriented receptive fields of different sizes (Hubel \& Wiesel, 1977). This organization indicates strongly that the mammalian visual system responds selectively to uniform orientation irrespective of the size of the elements that carry this stimulus property. Given the psychophysical evidence for orientational selectivity in the human

This research was supported by Grant A28115971R provided by the Australian Research Grants Scheme to the author. H. J. Jenkins is thanked for her research assistance and constructive criticism. The author's mailing address is: Department of Psychology, University of Western Australia, Nedlands, W.A. 6009, Australia. visual system (see Braddick, Campbell, \& Atkinson, 1978, for a review), it seems likely that a similar neural structure may comprise part of the human visual system.

Given this possibility, it may be a useful heuristic to consider using the known neurophysiology to account for the ability to perceive global patterns in bilaterally symmetric dot textures. Normally, these textures are described as they are above, as the reflection of a set of randomly placed elements across a given axis. This description seems to have dominated theoretical expositions with respect to perceiving symmetry in such textures, with one set of random points having to be compared with another at some stage in the visual system (e.g., Barlow \& Reeves, 1979; Corballis \& Beale, 1970; Julesz, 1971).

There is, however, an alternative means of describing bilaterally symmetric dot textures, one that is correlated to a greater extent with known mammalian neurophysiology: a bilaterally symmetric dot texture can also be described as a two-dimensional distribution of uniformly oriented point-pair elements, of nonuniform size, which fall across the same axis evenly such that the uniformly oriented pairs have collinear midpoints.

This description requires that the human visual system be capable of: (1) detecting the orientation of uniformly oriented point-pairs irrespective of their size, and (2) detecting that these element point-pairs have collinear midpoints.

The aim of this investigation was to determine the visual system's sensitivity to each of these factors.

With respect to the first, it is known that the visual 
system is capable of detecting orientational uniformity in dot textures comprising point-pairs of uniform size (e.g., Glass, 1969; Glass \& Switkes, 1976; Jenkins, 1983a). There is a linear spatial limit to the detectability of this orientational uniformity, which depends upon factors such as the texture size, its location on the retina, and its spatial frequency composition (Jenkins, 1983a), but it is unknown whether the visual system can detect orientational uniformity when the orientation is carried by point-pair elements varying in linear extent. The aim of Experiment 1 was to determine if the visual system had this capacity.

This was achieved by perturbing the midpoint collinearity of a bilaterally symmetric texture and comparing it with an equally dense random-dot texture. This procedure, while interfering with one of the two defining characteristics, leaves orientational uniformity unchanged; the uniformly oriented pointpair elements are simply spatially relocated with respect to each other. Therefore, if the visual system is sensitive to orientational uniformity, it should still be possible to discriminate the symmetric texture perturbed in this fashion from an equally dense randomdot texture.

Experiments 2 and 3 were conducted to determine the sensitivity of the visual system to the remaining factor, midpoint collinearity. The former experiment measured the ability of the visual system to detect the relative location of the midpoints of uniformly oriented point-pairs, and the latter measured its sensitivity to any deviation from perfect midpoint collinearity.

The aim of Experiment 2 was achieved by comparing two textures, each comprising uniformly oriented point-pairs of nonuniform size. In one, the midpoints of the point-pairs were randomly allocated over the horizontal extent of the texture, whereas in the other texture, the distribution of these midpoints progressed from collinearity over increasingly larger ranges about the central axis of the texture. If, at some point, the visual system loses the ability to detect relative location of uniformly oriented pointpair midpoints, discrimination between these two textures should break down.

Experiment 3 required the discrimination of a bilaterally symmetric texture (midpoint collinearity) from a texture comprising uniformly oriented pointpairs whose distribution of midpoints progressed from collinearity over increasingly larger ranges about the central axis of the texture. Discrimination would result, in this case, when the system detected a deviation from perfect collinearity.

A further experiment was conducted (Experiment 4) because the orientation of the axis of a bilaterally symmetric texture has been reported to be perceptually significant. Julesz (1971) observed that vertical axis symmetry was more perceptible than horizontal axis symmetry. In order to determine whether either or both of the suggested factors contributed to this orientational anisotropy, Experiments 1,2 , and 3 , which were conducted with vertical axis symmetry, were repeated, under the rubric of Experiment 4, with the symmetry about the horizontal axis.

Dynamic dot textures were used because the elements of this type of texture have no inherent structure or meaning. It follows that any correlation perceived in the texture is independent of the component elements. The texture is constructed by the rapid sequential plotting of uniformly oriented point-pairs, of different within-point-pair separations, but with collinear midpoints. An additional important advantage of this dynamic construction over a geometrically equivalent static form is that the probability of interference between component elements at a local level is reduced, since, in each stimulus stream, each element dot is physically present for only $1.5 \mu \mathrm{sec}$. Other advantages over the static texture include an even distribution of elements (no clustering) and a more perceptible stimulus under a wide range of viewing conditions (see Jenkins, 1983a).

\section{METHOD}

\section{Experimental Situation}

All experiments utilized dynamic dot textures plotted on a display oscilloscope under computer control. Subjects sat viewing the oscilloscope binocularly at a distance of $57.3 \mathrm{~cm}$. The textures were 5 deg square. The viewing distance was maintained by the use of a chin restraint for the subjects.

Stimulus luminance was $1.2 \log$ units above threshold throughout all experiments, with the oscilloscope face luminance maintained at $1 \mathrm{~cd} / \mathrm{m}^{2}$. For all experiments, the laboratory was dimly illuminated, so that the subjects did not undergo any radical change in visual sensitivity while waiting to participate in the experiments.

There was always sufficient illumination for the subjects to see the face of the oscilloscope, so that convergence and accommodation could be maintained at the appropriate distance. Each stimulus sequence was initiated by the subject, ensuring his attention to the screen at the appropriate moment.

\section{Apparatus}

The experiments were run under the control of a PDP-8/E computer, manufactured by the Digital Equipment Corporation. Interfaced to this computer was a fast-display oscilloscope, a Hewlett Packard 1332A oscilloscope equipped with an ultrashort P24 phosphor, for presentation of the stimuli. The intensity of a point plotted on this type of phosphor has a single peak with a maximum spectral-energy emission at $510 \mathrm{~nm}$, and the point fades to $10 \%$ of its original brightness in $1.5 \mu \mathrm{sec}$. It can therefore be considered that the effective physical duration of each point comprising the dynamic textures was approximately $1.5 \mu \mathrm{sec}$.

\section{Generation of Stimuli}

The dynamic textures were obtained by the rapid sequential plotting of points on an oscilloscope face within a square region, $9 \times 9 \mathrm{~cm}$, occluded by a mask to $5 \times 5 \mathrm{~cm}$. This region was a 256 $\times 256$ matrix, so that the location of any point was given by one of 256 possible $x$ values and one of 256 possibly $y$ values.

A single random point could be plotted by obtaining a random number between 0 and 255 for $x$ and, similarly, a random number for $y$. The numbers were obtained using the computer's randomnumber generator. These two coordinates, together with an in- 
tensity value, were then inserted into the appropriate locations in a computer plotting subroutine. The intensity value was held constant during all experiments. Once these values had been inserted, the next section in the program plotted the point at the specific coordinates on the oscilloscope screen.

\section{Dynamic Symmetric Textures}

The vertical-axis fields were constructed by the sequential plotting of independent collinear point-pairs with horizontal orientation, with the position of each new point-pair being independent of every other point-pair along the vertical axis. The position of each horizontal point-pair was determined in the same manner: the $x$ and $y$ values of a random point on either side of a vertical axis were selected independently of each other; then the $x$ value was reflected. This new $x$ value, together with the original $y$ value, specified the coordinates of the partner point. Both points were then plotted together, with a duration of $1.5 \mu \mathrm{sec}$, and there was an interval of $121.6 \mu \mathrm{sec}$ between the plotting of successive independent point-pairs. With continuous rapid sequential plotting, the result was a texture that appeared to be bilaterally symmetric, that is, with one half of the texture being reflected about a central vertical axis. For horizontal axis construction, the process was rotated through $90 \mathrm{deg}$.

\section{Distribution of Point-Pair midpoints}

The above construction ensured that the midpoints of all identically oriented point-pairs were collinear along a vertical line, the axis of symmetry; that is, the midpoints were constrained to exactly the same $x$ coordinate, with the point-pair $y$ coordinate randomly distributed (and vice versa for symmetry about the horizontal axis). To discompose this axis, it was possible to arrange for midpoints to fall at random within a given range about this $x$ coordinate. This region of midpoint distribution was always centered about the axis of symmetry. The distribution of midpoints within these regions was random.

\section{Dynamic Noise Textures.}

The dynamic noise textures were constructed by the sequential plotting of independent random points, with the position of each new point independent of every other point. Two random points were obtained and plotted together, with a duration of $1.5 \mu \mathrm{sec}$, and there was an interval of $121.6 \mu \mathrm{sec}$ between successive pairs of random points.

\section{Perceived Dot Denulty}

The method of constructing dynamic textures meant that in order to present a texture with a duration of $1 \mathrm{sec}$ at this plotting rate of two new points every $121.6 \mu \mathrm{sec}$, the computer had to plot 16,446 points $(8,223$ sequential pairs of points).

These points were distributed over the oscilloscope face $(9 \times 9 \mathrm{~cm})$ so that the distribution of points was approximately 203 points per square centimeter over the 1-sec interval. The perceived density of the resulting texture was approximately 26 points per square degree of visual angle, assuming a temporal integration period of $130 \mathrm{msec}$ for this type of stimulus (see Jenkins, 1983a).

It must be emphasized that the dynamic textures comprise a continuous train of independent pairs of points, so that at any instant only two points are physically present on the screen. The estimate of perceived density arises from the continuous nature of this train, and not from any consideration of "frame" or frame rate. For all experiments, the perceived density was kept constant at approximately 26 points per square degree of visual angle.

\section{Experimental Procedure}

The psychophysical method used was a two-alternative temporal forced-choice technique, in which each trial comprised the presentation of two textures, the standard and the experimental stimulus. Each session comprised 100 trials, 20 trials of each of five values of the independent variable presented randomly. The order of stimulus location within each trial was also random. Five sessions were completed by each subject for each experimental condition.

To initiate a trial, the subject depressed one of two hand-held pushbuttons, after which the stimulus sequence commenced immediately. Two textures appeared on the screen sequentially, each of $1 \mathrm{sec}$ duration and each separated from the other by a 1-sec interstimulus interval. The subject then identified the temporal location of the stimulus of interest and pressed the pushbutton corresponding to "first" or "second" as appropriate. This decision was signaled to the computer, and display parameters were then computed for the next trial (computing time was approximately $10 \mathrm{msec}$ ); the subject was then free to initiate the next trial. There was no pressure to complete a session within a given time limit, but, in practice, it was found that each set of 100 trials was completed in approximately $15 \mathrm{~min}$. At the conclusion of each session, the computer printed out the results on the teletypewriter.

\section{Subjects}

The sequence of events outlined in the preceding section was conducted with three observers for each experiment. All had normal or corrected-to-normal vision. All observers were practiced in vision research and with the method used, but all were naive with respect to the aims of the experiments.

\section{RESULTS}

\section{Experiment 1}

The experimental stimulus was a bilaterally symmetric dynamic dot texture whose point-pair midpoints were distributed randomly within one of five possible ranges centered about the axis of symmetry: 0.0 (collinear midpoints), $0.49,1.05,2.17$, and 4.41 deg of visual angle. The choice of these values was determined by technical considerations and by the number system used by the computer's assembly language. The axis of symmetry was vertical. The standard stimulus was an equally dense random-dot texture, and the subject's task was to determine which of the two was the symmetric stimulus.

The results of Experiment 1 are illustrated in Figure 1 and show that, for all subjects, discrimination was perfect with no midpoint perturbation but that, as the midpoints were distributed randomly over a range centered about the axis of symmetry, perfect discrimination broke down. It should be noted, however, that, for all subjects, discrimination never fell below $65 \%$ correct, even with the most extreme distribution (4.41 deg of visual angle). In addition, the performance curves for all subjects have the appearance of composite curves, with discrimination declining fairly rapidly to a midpoint distribution of $2.17 \mathrm{deg}$ of visual angle and leveling out at that point.

\section{Experiment 2}

The experimental stimulus was identical to that used in Experiment 1, with the same range of midpoint distributions. The standard stimulus was constructed in an identical manner to the experimental stimulus, but the midpoints of each point-pair were situated at random over a range as wide as the stim- 


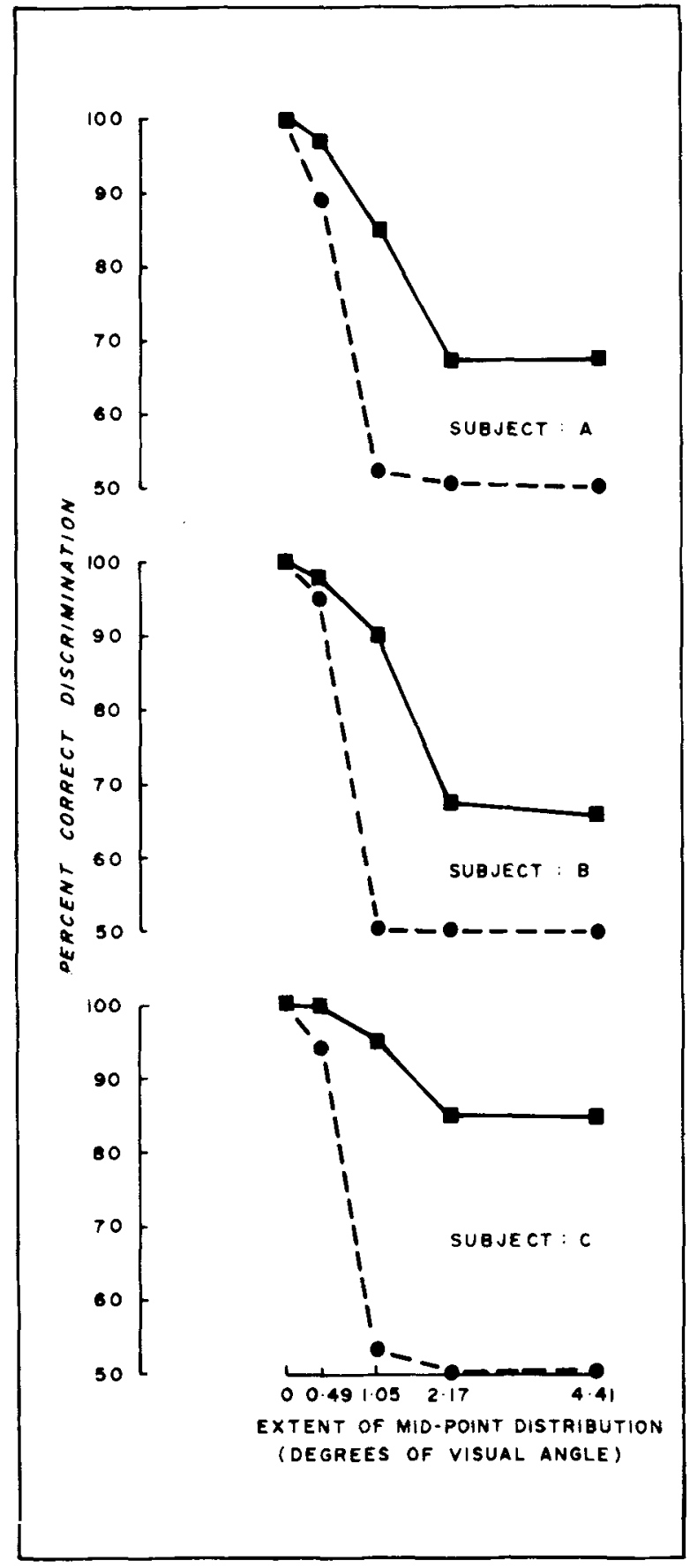

Figure 1. Percent correct discrimination is shown for Experiment 1 , the detection of orientational uniformity (solid line), and Experiment 2, the detection of midpoint collinearity (dashed line). Results are shown for three subjects.

ulus texture (in this case, $5 \mathrm{deg}$ of visual angle). The subject's task was to detect the stimulus texture that most nearly approximated perfect symmetry.

Figure 1 also illustrates the results of Experiment 2, and shows that, for all subjects, discrimination was perfect with no midpoint redistribution.
However, as these midpoints were distributed over a wider range about the axis of symmetry, discrimination declined rapidly, reaching chance at $1.05 \mathrm{deg}$ of visual angle.

\section{Experiment 3}

The experimental stimulus was the same as that used in Experiments 1 and 2, except that the range of midpoint distributions was smaller; $0.0,0.07,0.21$, 0.49 , and $1.05 \mathrm{deg}$ of visual angle. The standard stimulus was an identical stimulus, with collinear midpoints. The subject's task was to detect the perfectly symmetric stimulus texture.

The results of Experiment 3, shown in Figure 2, demonstrate that for all subjects once the midpoints of the uniformly oriented point-pairs were distributed over a region of $0.21 \mathrm{deg}$ of visual angle, discrimination was perfect. It should be noted, however, that, with perturbation of midpoints over a range as small as $0.07 \mathrm{deg}$ of visual angle, the visual system can detect this deviation with a probability greater than chance.

\section{Experiment 4}

Experiments 1, 2, and 3 were repeated with the symmetry constructed about a horizontal axis.

Figure 3 shows the results of Experiments 1 and 2 for two different orientations. It appears that, for all subjects, discrimination in Experiment 1 was superior when the symmetry was about the horizontal axis. The qualitative similarity of the performance curves should also be noted, in addition to quantitative variations. There appears to be no consistent ad-

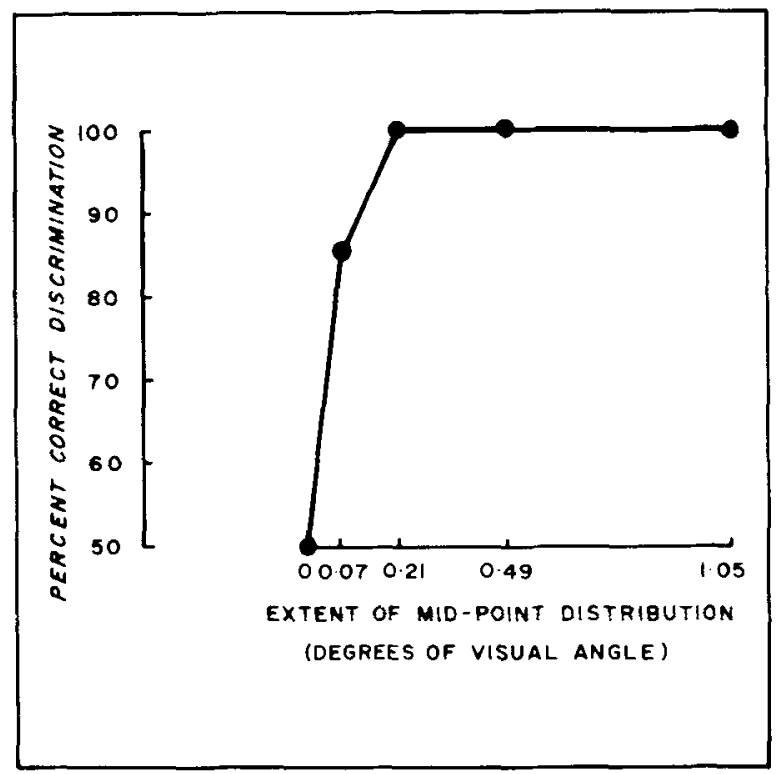

Figure 2. Percent correct discrimination is shown for Experiment 3 , for three subjects. The detection of deviation from perfect midpoint collinearity is Identical for the three subjects. 


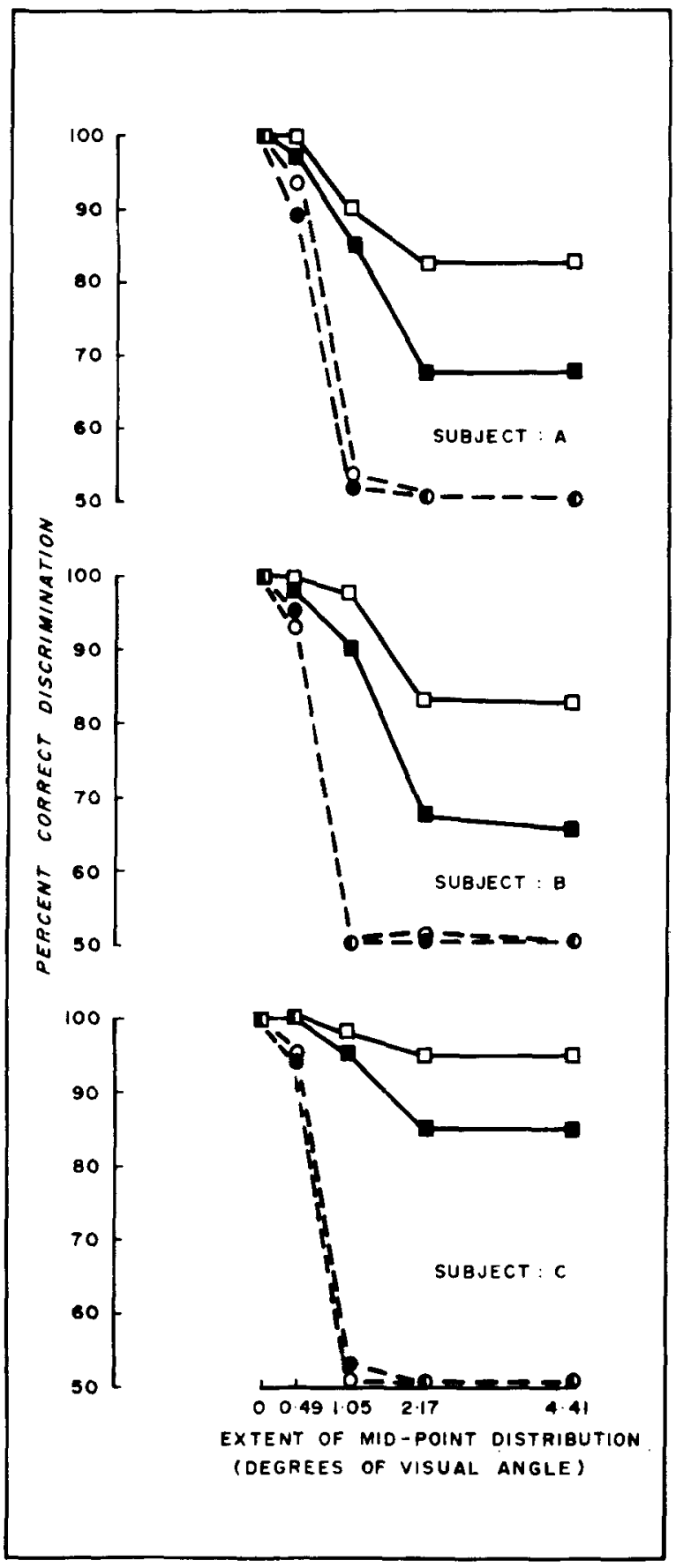

Figure 3. Percent correct dlecrimination is shown for Experments 1 (solid line) and 2 (dushed line) for two orientation, verthcal (colld aymboly) and horkzontal (hollow symbols).

vantage of orientation with respect to the detection of midpoint location in Experiment 2.

Figure 4 illustrates the results of Experiment 3 for both orientations, and it can be seen, for all subjects, that perturbation of midpoint collinearity was more easily detected when the axis of symmetry was vertical. Discrimination was perfect in the horizontal case, with a distribution range of $0.49 \mathrm{deg}$, which is more than a factor of two greater than the vertical case.

\section{DISCUSSION}

The aim of this investigation was, first, to determine whether the visual system could detect uni-

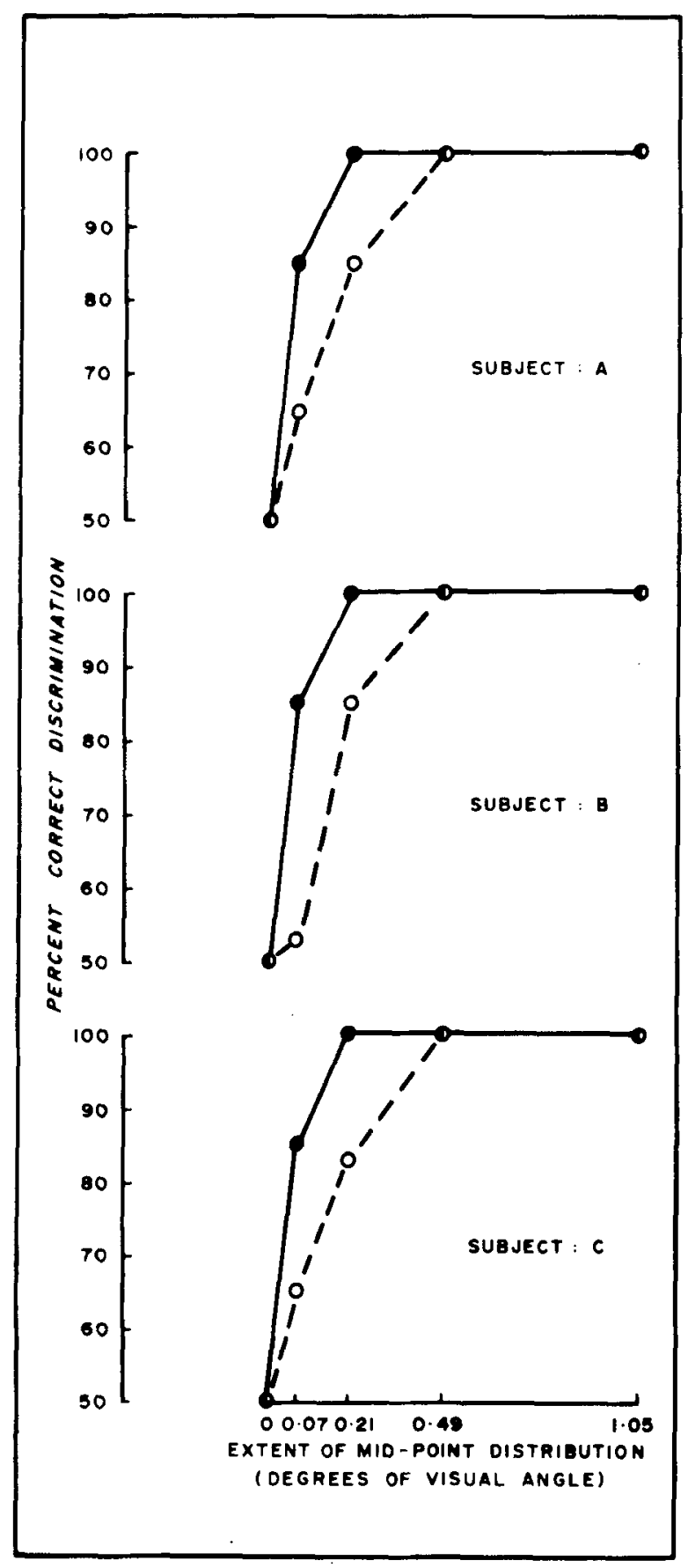

Figure 4. Percent correct dbcriminntion in showe tor Bxperiment 3 for two orfentations, vertical (colid live, solid ajmbob) and horkoutal (dashed Hee, hollow oymbole). 
formly oriented point-pairs irrespective of their size, and second, whether it could then determine the relative location of the midpoints of these element point-pairs.

\section{Orientational Uniformity}

Experiment 1 demonstrated that if the midpoints of the uniformly oriented point-pairs comprising a bilaterally symmetric texture are distributed randomly over a region almost as wide as the texture, it is still possible to discriminate such a pattern from an equally dense random dot texture. Since Experiment 2 demonstrated that beyond a distribution range of approximately $1.0 \mathrm{deg}$ of visual angle, the distribution might as well be random, the stimulus characteristic providing the basis for the discrimination beyond this point in Experiment 1 was probably the uniform orientation of the element point-pairs, since all other factors were equal. Subjects did report that on some trials, the striated appearance of one of the textures provided evidence that it was not a random texture.

Further support for this conclusion is provided by the difference in discriminability for different orientations in Experiment 1 (see Figure 4). Discrimination was superior when the symmetry was about the horizontal axis, that is, when the uniformly oriented point-pairs were vertical. It has been found that the human visual system is more sensitive to uniformly oriented point-pairs of uniform size if they are vertical (Jenkins, Note 1). ${ }^{1}$ It is interesting to note that the orientational anisotropy exists for textures comprising orientationally uniform point-pairs of uniform size and orientationally uniform textures of nonuniform size. The fact that discrimination is possible independently of the distribution of point-pair sizes supports the arguments of Glass (1969) and Jenkins (1983a) that the detection of orientational uniformity in point-pair textures by the human visual system could be due to the existence of cells in Area 17 of the visual cortex, similar in structure and function to those revealed in the mammalian visual cortex (see Hubel \& Wiesel, 1977).

\section{Collinearity of Midpoints}

Two aspects of the ability of the visual system to locate the midpoints of uniformly oriented pointpairs were investigated: (1) the midpoint perturbation beyond which the visual system could not correlate the element point-pairs on any characteristic other than orientational uniformity (Experiment 2) and (2) the midpoint perturbation required in order to be detected as deviating from perfect collinearity (Experiment 3).

With respect to the first aspect, once the midpoints were distributed randomly over a region larger than approximately $1.0 \mathrm{deg}$ of visual angle, the visual system was unable to correlate them, since, beyond this point, such a texture was indiscriminable from a similar texture whose midpoints were randomly distributed over 5 deg of visual angle (the horizontal extent of the pattern). Within the 1-deg region, how does the visual system correlate midpoints? Midpoints are intangible entities, and geometrically it would be necessary to identify the horizontal boundaries of each pair of uniformly oriented points in order to locate the midpoint. This problem has been limited in one sense by Jenkins (1983b), who found that point-pairs greater than $1.0 \mathrm{deg}$ of visual angle were redundant in the perceptibility of symmetry in these textures. So the question remains: How does the visual system correlate midpoints of pairs less than $1 \mathrm{deg}$ of visual angle? A possible clue is provided by subjects who reported that the discrimination between the textures was based on the presence or absence of a central coherent feature. The subjects' reports indicated that with a midpoint distribution of $\sim 0.5 \mathrm{deg}$ of visual angle, this feature was always present, but that beyond a distribution of approximately $1.0 \mathrm{deg}$, the feature did not appear.

This observation, together with the results of Experiment 2, suggests that there is a fusional process which combines constituent elements into a coherent whole, a segregated identifiable feature, based on principles similar to those described by Olson and Attneave (1970). The most likely candidates for the constituent elements are the smaller, most salient, least redundant individual point-pairs identified by Jenkins (1982) as subtending approximately $0.3 \mathrm{deg}$ of arc. These are postulated because they are similar in size and orientation, and they fall within a restricted spatial region of the symmetric texture. The basis for the salience of the smaller point-pairs is not known, but other areas of investigation suggest that there is some differentiation in the visual system when dealing with stimulation above and below approximately $0.5 \mathrm{deg}$ of visual angle (Jenkins, 1983b; Ross, Jenkins, \& Johnstone, 1980).

The second aspect of collinearity perturbation, the detection of deviation from perfect midpoint collinearity, reveals quite a different process, with subjects reporting that it was based on whether or not the coherent feature was symmetrical. The sensitivity illustrated in Experiment 3 confirms that the slightest deviation from perfect symmetry is detected. In this case, if the point-pair midpoints are distributed over a region of $0.04 \mathrm{deg}$ of visual angle wide $(2.4 \mathrm{~min})$, then $75 \%$ correct discrimination occurs. By extrapolation of the slope of the curve shown in Figure 2 , this would reach $100 \%$ correct with a distribution of $0.1 \mathrm{deg}$ of visual angle $(6 \mathrm{~min})$. This is a remarkable resolution and very close to the two-point threshold for fields comprising identical point-pairs (approximately $2 \mathrm{~min}$ of arc). Therefore, this process is probably the same as that which Mach (1886) considered so important to the problem of form vision, 
and which has been investigated subsequently using a wide variety of stimuli, such as geometric shapes and letters (e.g., Bradshaw, Bradley, \& Patterson, 1976; Corballis, Miller, \& Morgan, 1971; Sekuler \& Pierce, 1973; Staller \& Sekuler, 1977).

\section{Component Processes}

The results of Experiments 1, 2, and 3 suggest that there are three distinct processes involved when the human visual system is presented with a bilaterally symmetric dot texture: first, a process that detects the orientational uniformity of the component pointpairs, irrespective of their size; second, a process that fuses the most salient point-pairs into a salient feature; and third, a process that determines whether this feature is symmetric.

Further support for these three separate processes is provided by the results of Experiment 4, which showed that each of the proposed component processes responds differently to variation in the orientation of the axis of symmetry. The orientational uniformity process is most sensitive when the axis is horizontal, that is, when the uniform orientation of the point-pairs is vertical. This is consistent with the case of transpositional symmetry, in which the pointpairs have not only uniform orientation, but also uniform size (see Jenkins, Note 1, and Footnote 1). The fusional process is indifferent to orientation, whereas the third process, that which detects whether features or objects are symmetrical, seems to prefer the vertical axis. This observation of vertical preference, first noted by Mach (1886), is also consistent with subsequent experimental findings with respect to this particular attribute (e.g., Corballis \& Roldan, 1975; Palmer \& Hemenway, 1978).

\section{Interaction of the Component Processes}

Given the existence of three distinct processes, questions immediately arise as to their locus and their possible interaction. With respect to the first process, the detection of orientational uniformity, Glass (1969) and Jenkins (1983a) have argued that it occurs in Area 17 of the visual cortex, since, in the mammalian visual cortex, this is the area that contains the neural machinery capable of detecting orientational uniformity. It is difficult to locate the other two processes, but it is probable that the second precedes the third, since this latter process is detecting a characteristic of an object or feature produced by the second. It must be noted, however, that despite the attractive rationality of such an argument, it is not logically impossible that an attribute of an object could be processed prior to the object itself.

Evidence for interaction is provided by two observations. First, when viewing a bilaterally symmetric texture, the salience of the central feature is dominant, and the striated structure, which is reported by observers when midpoints are distributed over an area greater than 1 deg of visual angle, is not perceived. This suggests that the product of the fusional process, or the process itself, masks the detection of orientational uniformity.

The second observation providing evidence for interaction between the component processes is that vertical axis symmetry is more perceptible than horizontal axis symmetry. Experiment 4 (see Figure 3) showed that the process of detecting orientational uniformity is superior when the axis of symmetry is horizontal and the midpoint collinearity is perturbed, yet this is of no apparent benefit in the final outcome, when all processes are acting together.

\section{Theoretical Implications}

The major implication of the results of these experiments is that it is unnecessary to postulate the existence of a symmetrical neural organization centered about the fovea to account for the detectability of structure in bilaterally symmetric textures. The three processes described-one that detects orientational uniformity, the fusional process, and the process that detects the symmetry of a feature-together can account for the immediate response of the visual system, as they can for the reported orientational anisotropy.

There is a further observation in favor of the hypothesized point-by-point analysis about the fovea reported by Julesz (1971), with experimental support provided by Barlow and Reeves (1979): the detection of symmetry in a bilaterally symmetric texture is impaired if the pattern is viewed eccentrically, off the fovea. Can this observation be accounted for by the alternative component-process hypothesis? Each of the three proposed processes may operate differentially across the retina. The detection of orientational uniformity is enhanced when viewed eccentrically (Jenkins, 1983a), and the detection of the symmetry of objects is independent of retinal locus-that is, a symmetrical object still looks symmetrical even if viewed eccentrically (Julesz, 1971). It is not known whether the fusional process changes across the retina, but if two-point resolution is important to this process, then it would certainly be affected by retinal locus, since two-point resolution is known to decline as a function of retinal eccentricity. Since the orientational uniformity process has been shown to be weak in interaction, the possibility that the other two processes together can account for the decline in detectability must be considered; for example, if the fusion of resolvable point-pairs declines with eccentricity, then one would expect that the formation of the salient feature would also be impaired. It may be, therefore, that eccentrically the problem may not be in detecting symmetry, but in detecting the object, or feature. This possibility is currently being investigated.

In summary, the results indicate that it is not nec- 
essary to postulate the existence of a symmetric neural structure to account for the perceptibility of bilaterally symmetric dot textures. An alternative proposition, supported by the data, is that three component processes, independently, and in interaction, may be involved in the perception of bilaterally symmetric dot textures.

\section{REFERENCE NOTE}

1. Jenkins, B. Orientational anisotropy in the detection of correlations. Manuscript in preparation, 1983.

\section{REFERENCWS}

Barlow, H. B., \& ReEve8, B. C. The versatility and absolute efficiency of detecting mirror symmetry in random dot displays. $V i$ sion Research, 1979, 19, 783-793.

Braddick, O., Campbell, F. W., \& Atrinson, J. Channels in vision: Basic aspects. In R. Held, H. Leibowitz, \& H. L. Teuber (Eds.), Handbook of sensory physiology (Vol. 8); perception. Heidelberg: Springer-Verlag, 1978.

Bradshaw, J., Bradley, D., \& Patterson, K. The perception and identification of mirror-reversed patterns. Quarterly Journal of Experimental Psychology, 1976, 28, 221-246.

Corballis, M. C., \& Benle, I. L. Bilateral symmetry and behaviour. Psychological Review, 1970, 77, 451-464.

Corballis, M. C., Miller, A., \& Morgan, M. J. The role of left-right orientation in interhemispheric matching of visual information. Perception \& Psychophysics, 1971, 10, 385-388.

Corballis, M. C., \& Roldan, C. E. Detection of symmetry as a function of angular orientation. Journal of Experimental Psychology: Human Perception and Performance, 1975, 1, 221-230.

Guass, L. Moiré effects from random dots. Nature, 1969, 233, 578-580.

Grass, L., \& Switkes, E. Pattern recognition in humans: Correlations which cannot be perceived. Perception, 1976, 5, 67-72.

Hubet, D., \& Wiesel, T. N. Ferrier lecture: Functional architecture of Macaque monkey visual cortex. Proceedings of the Royal Society, London, Series B, 1977, 198, 1-59.
Jenkins, B. Redundancy in the perception of bilateral symmetry in dot textures. Perception \& Psychophysics, 1982, 32, 171-177.

JEnkins, B. Spatial limits to the detection of transpositional symmetry in dynamic dot textures. Journal of Experimental Psychology: Human Perception and Performance, 1983, 9, 258-269. (a)

Jenkins, B. Temporal limits to the detection of correlation in transpositionally symmettic textures. Perception \& Psychophysics, 1983, 33, 79-84. (b)

Julesz, B. Foundations of cyclopean perception. Chicago: Chicago University Press, 1971.

МАсн, Е. The analysis of sensations and the relation of the physical to the psychical. New York: Dover, 1959. (Originally published in German, 1886).

Olson, R. K., \& ATtnenve, F. What variables produce similarity grouping? American Journal of Psychology, 1970, 83, $1-21$

Palmer, S. E., \& Hemenway, K. Orientation and symmetry: Effects of multiple rotational and near symmetries. Journal of Experimental Psychology: Human Perception and Performance, 1978, 4, 691-702.

Ross, J., Jenkins, B., \& Johnstone, J. R. Size constancy fails below half a degree. Nature, 1980, 283, 473-474.

Sekuler, R., \& Pierce, S. Perception of stimulus direction: Hemisphere homology and laterality. American Journal of Psychology, 1973, 86, 679-695.

Staller, J., \& Sekuler, R. Stimulus and response factors in mirror image discrimination. Perception \& Psychophysics, $1977,22,592-598$.

\section{NOTE}

1. It has been found that the spatial tolerance (the upper limit) for perceiving structure in transpositionally symmetric textures varies as a function of the orientation of the symmetry. Vertical upper limits are consistently larger than oblique or horizontal upper limits. There is no consistent difference between the latter two orientations (Jenkins, Note 1).

(Manuscript received November 16, 1982; revision accepted for publication July 8,1983 .) 